Article

\title{
Development of a Lightweight Prosthetic Hand for Patients with Amputated Fingers
}

\author{
Wooseok Ryu ${ }^{1}\left(\right.$, Youngjin Choi ${ }^{2}{ }^{(D)}$, Yong Je Choi $^{1}$ and Sungon Lee ${ }^{2, *}$ \\ 1 Department of Mechanical Engineering, Yonsei University, Seoul 03722, Korea; fbdntjr3@gmail.com (W.R.); \\ yjchoi@yonsei.ac.kr (Y.J.C.) \\ 2 School of Electrical and Engineering, Hanyang University, Ansan 15588, Korea; cyj@hanyang.ac.kr \\ * Correspondence: sungon@hanyang.ac.kr; Tel.: +82-31-400-5174
}

Received: 27 April 2020; Accepted: 16 May 2020; Published: 20 May 2020

Featured Application: We developed a lightweight active prosthetic hand using 3D printing technology with nylon (weight: $\mathbf{1 5 2 . 3 2}$ g). Patients with amputated fingers, especially amputees with a thumb and metacarpal, can use this cost-effective lightweight prosthetic hand comfortably.

\begin{abstract}
Finger amputations are the most common upper limb amputation, and they occur approximately 100 times more often than hand amputations. We developed a prosthetic hand for amputees with a thumb and metacarpal. In this case of amputation, the installation of actuators and electrical components is difficult because space is considerably limited owing to the residual metacarpal. This design issue is solved by installing actuators vertically between finger modules and the mounting part where the electrical parts are embedded. With this design, the arrangement between the palm of the patient and the fingers of the prosthetic hand can be adjusted as well. Weight is also an especially important design issue in the development of a practical prosthetic hand because the patient perceives that weight. Most prosthetic hands are attached to the residual limb of the amputee by compressing the contact surface between the product and the amputated body part of the patient. Heavy weight causes users to feel discomfort and fatigue over extended periods of usage. In this study, all frames used in the proposed prosthetic hand were fabricated from nylon using multi-jet-fusion three-dimensional printing. As a result, the weight of the developed prosthetic hand was only $152.32 \mathrm{~g}$ but still had the desired strength and stiffness. Each prosthetic finger has four-degree-of-freedom. The distal interphalangeal, proximal interphalangeal, and metacarpophalangeal joints are coupled and driven by power from an actuator, which is transferred to each joint through the tendons. Adduction, abduction, and self-adaptive grasping motions were passively realized using linear and torsional springs. The prototype was fabricated based on these design concepts and functions, and its functionality was verified in experiments using diverse objects.
\end{abstract}

Keywords: finger amputation; lightweight prosthetic hand;3D printing; tendon; self-adaptive grasping

\section{Introduction}

For amputees who have lost their fingers or hands in accidents or because of complications, various dexterous prosthetic hands (or fingers) have been designed. Such prosthetic hands can be classified into three types according to the amputated patterns and levels of patients: Type 1 (with middle or proximal phalanx), Type 2 (with only a partial carpal), and Type 3 (with thumb and partial metacarpal) [1-3].

Prosthetic hands for patients of Type 1 have been designed as finger modules that consist of a linkage mechanism driven by the patient's body because the proximal interphalangeal (PIP) or metacarpophalangeal (MCP) joints can be moved by the patients. The linkage mechanisms used in 
these prosthetic fingers (by Rodriguez et al. and by Yoon et al.) can achieve flexion and extension motions $[4,5]$. In particular, the self-adaptive finger designed by Yoon et al. consists of a linkage mechanism that can be driven using body power by patients with partial proximal phalanx motion.

In Type 2, actuators should be installed in prosthetic hands to produce finger motions because there are no sources of body power. Most actuators are installed in the palm of the prosthetic hand. An under-actuated five-finger prosthetic hand was developed (by Kamikawa) for grasping, and fifteen compliant joints were driven using only one ultrasonic motor [6]. Jing developed a biomimetic prosthetic hand with the aim of reducing weight and cost by using two actuators: one to produce flexion/extension of the five digits and the other to generate adduction/abduction of the thumb [7]. In addition, the under-actuated tendon-driven prosthetic hand and the Bebionic hand (by RSL Steeper) have been developed, and the specifications are summarized in Table $1[8,9]$.

Table 1. Specifications of the existing prosthetic/robotic hands and prosthetic hand developed in this study.

\begin{tabular}{cccccccc}
\hline Developer [Ref.] & Type & Weight (g) & $\begin{array}{c}\text { Overall Size (mm) } \\
\text { [Length, Width, } \\
\text { Thickness] }\end{array}$ & $\begin{array}{c}\text { Number } \\
\text { of Joints }\end{array}$ & $\begin{array}{c}\text { Number } \\
\text { of } \\
\text { Actuators }\end{array}$ & $\begin{array}{c}\text { Joint } \\
\text { Coupling } \\
\text { Method }\end{array}$ & $\begin{array}{c}\text { Adaptive } \\
\text { Grasping }\end{array}$ \\
\hline Kamikawa [6] & 2 & 730 & {$[320,80-90,20]$} & 15 & 1 & $\begin{array}{c}\text { Tendon \& } \\
\text { linkage }\end{array}$ & Yes \\
\hline Jing [7] & 2 & 250 & - & - & 2 & Tendon & Yes \\
\hline Mottard [8] & 2 & $\begin{array}{c}298 \text { (Except } \\
\text { actuators) }\end{array}$ & - & 14 & 2 & Tendon & Yes \\
\hline RSL Steeper [9] & 2 & $495-539$ & {$[190-200,84-92,50]$} & 11 & 5 & Linkage & Yes \\
\hline Touch Bionics [10] & 3 & $450-615$ & {$[180-182,75-80$,} & 11 & 5 & Tendon & Yes \\
\hline Ryu [11] & 3 & 320 & {$[120,75]$} & $16,13]$ & 4 & Tendon & Yes \\
\hline This study & 3 & $\begin{array}{c}152.32 \\
\text { (Except thumb) }\end{array}$ & {$[174,77,11]$} & 18 & 4 & Tendon & Yes \\
\hline
\end{tabular}

In comparison with the above two types, the development of prosthetic hands for patients of Type 3 is difficult because the available space for installing actuators is limited, and sources of body power do not exist. I-limb digits (by Touch Bionics) is a commercialized prosthetic hand for patients with a partial metacarpal [10]. The device consists of two joints in each finger and is driven by an actuator. Although it is compact and includes electrical components, it cannot perform self-adaptive grasping and adduction/abduction motions. In addition, a prosthetic finger with an actuator embedded in the proximal phalanx has been developed to eliminate the mounting part [11]. However, the finger does not have the space to install the electrical components. Additionally, diverse prosthetic hands have been developed $[12,13]$.

In this study, a prosthetic hand was developed to realize self-adaptive grasping, adduction/ abduction, and extension/flexion motions, as shown in Figure 1. The design concepts were focused on the development of a lightweight, practical hand for amputees of Type 3 . The finger module was designed using an under-actuated mechanism with four-degree-of-freedom (4-DOFs). The three revolute joints of the finger corresponding to the distal interphalangeal (DIP), PIP, and an axis of MCP joints are coupled and driven by tendons, which transfer power produced by an actuator to each joint. Moreover, by using three-dimensional (3D) printing technology, a lightweight prosthetic hand was developed.

This paper is organized as follows: The design concepts and 3D models of the suggested prosthetic hand are described and illustrated in Section 2. In Section 3, the kinematic, static, and finite element method (FEM) analyses of the proposed prosthetic hand are explained. Finally, in Section 4, the prototype hand is represented, and experiments are performed. 


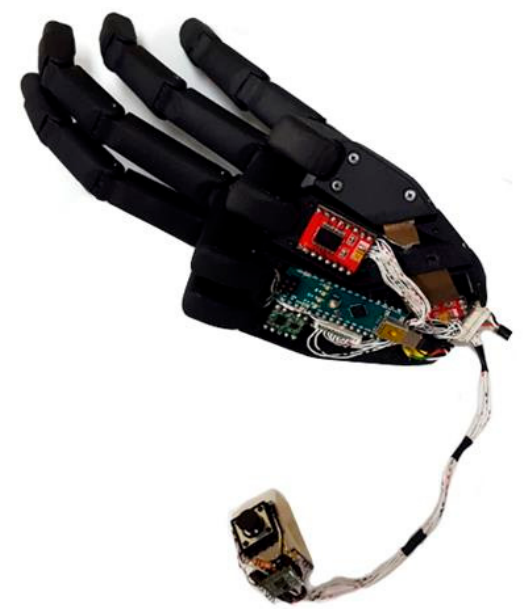

(a)

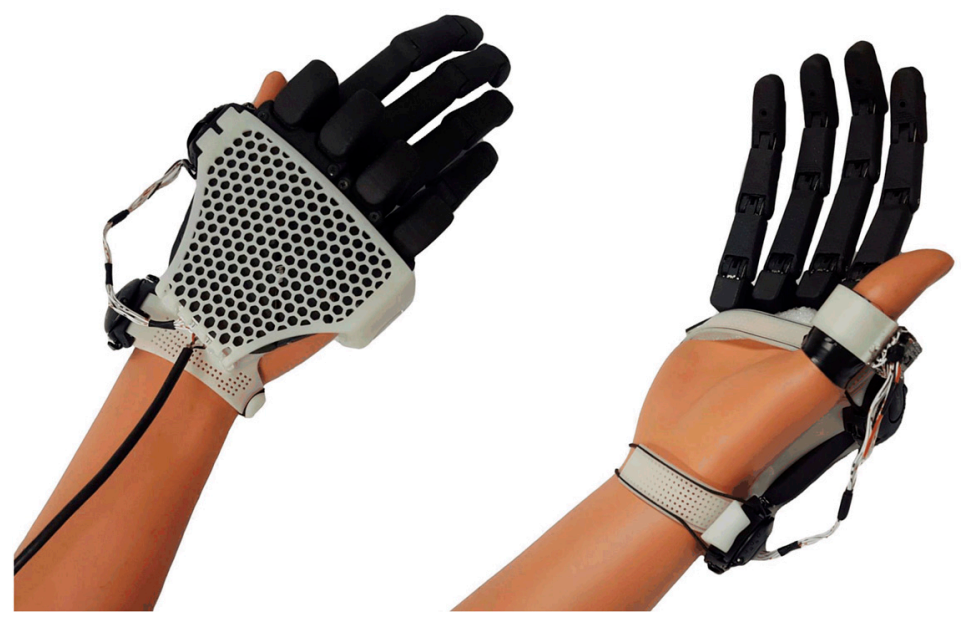

(b)

Figure 1. (a) Developed prosthetic hand and (b) hand with prosthesis.

\section{Design of the Prosthetic Hand}

\subsection{Design Concepts and 3D Models}

To develop a prosthetic hand for amputees who have retained their thumb and partial metacarpal, we considered the following design concepts. First, the mounting part was designed with the minimum thickness $(11 \mathrm{~mm})$ to include the required electrical components, for example, motor drives, a microcontroller unit (MCU), and a sensor. Then, the actuators were installed vertically between the finger modules and the mounting part, as shown in Figure 2a. This configuration allowed for the installation of actuators despite the limited available space caused by the presence of the residual metacarpal. Moreover, the arrangement between the patient's palm and the fingers of the prosthetic hand can be adjusted by modifying the length $(L)$ of the vertical frame shown in Figure 2a. Second, the product weight was reduced by manufacturing all frames with nylon by using Multi-Jet-Fusion (MJF) 3D printing, except for the bearings, shafts, springs, and tendon-fixing frame. Small-sized parts fabricated using the MJF method offer superior strength and durability than those fabricated using other methods $[14,15]$. Consequently, this 3D printing technology is suitable for fabricating parts of the prosthetic hand. Third, each finger was designed as a module. Therefore, finger modules can be attached and detached easily during the assembly process, depending on the configuration of the amputated fingers. In each finger module, driving power is produced using a direct current (DC) motor and transferred to the joint frames in the distal, middle, and proximal phalanxes through tendons, which are depicted with red and green lines in Figure 2a. The tendons are connected to the actuator by means of a tendon-fixing frame. Fourth, the prosthetic hand can be fixed on the back of the user's hand by using two dials (Figure 1b). Finally, if the mounting part is designed as a single frame, it will cause user discomfort when objects are grasped because the frame cannot produce movements similar to those of the palm. Consequently, the mounting part of the proposed hand was designed with 2-DOFs and moved passively by using leaf springs (Figure 2b). The second to the fifth concepts are focused on practicality.

The desired weight of the prosthetic hand was set to be $<160 \mathrm{~g}$, which is $40 \%$ of the suitable weight of the prosthetic hand suggested in [16]. The target length and width were determined to be 173 and $79 \mathrm{~mm}$, respectively, based on anthropometric dimensional data for American female hands [17]. In extension and flexion motions (natural motion), the desired velocity of the distal phalanx was $>180^{\circ} / \mathrm{s}$. Moreover, the payload at the fingertip of a finger module was computed to be $>250 \mathrm{~g}$, which is adequate to lift an object weighing $1 \mathrm{~kg}$ by using four fingers, and the product cost was <250 USD. The ranges of each phalanx in natural motion, of self-adaptive grasping by the middle and proximal phalanxes, of the adduction/abduction motions, and of each joint for palm motion are $0^{\circ}-90^{\circ}, 0^{\circ}-25^{\circ}$, 
$0^{\circ}-15^{\circ}$, and $0^{\circ}-15^{\circ}$, respectively. Furthermore, the target grasping motions include holding objects such as paper cups, pens, balls of various sizes, tools, and smartphones. To realize grasping motion, the figure module was designed as an under-actuated mechanism with 1-DOF active and 3-DOFs passive motions. In the proposed finger module, the DIP joint is driven directly. In the case of the PIP and MCP joints, although the joint frames in Figure 2a are directly connected to the tendon-fixing frame, the motions of the PIP (1-DOF) and MCP (2-DOFs) joints are realized passively by using linear and torsional springs. The prosthetic hand based on these design concepts and mechanisms can achieve self-adaptive grasping according to object shapes, extension/flexion and adduction/abduction motions.

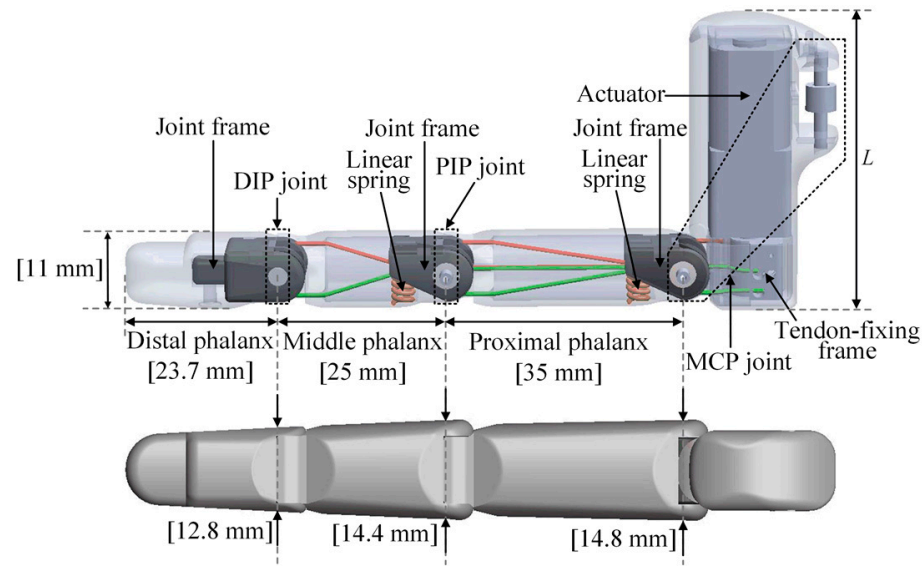

(a)

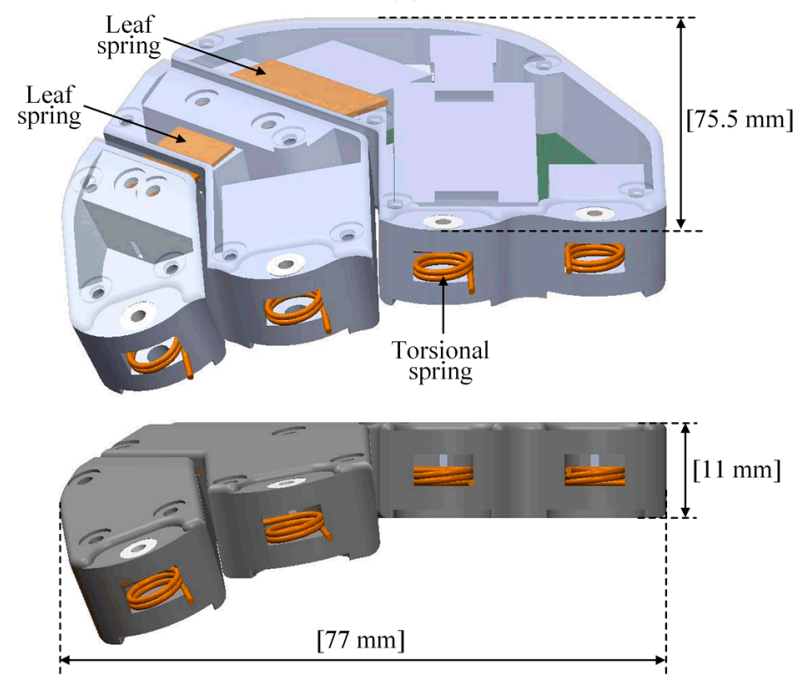

(b)

Figure 2. Nomenclature of components and frame sizes in (a) finger module and (b) mounting part.

\subsection{Principles of Operation}

Each phalanx is moved using tendon-driven method, as shown in Figure 3. The joint frames in each phalanx are connected by tendons attached to the tendon-fixing frame. The ends of each tendon are fixed (as shown by the red and green points) on each joint frame, and the tendons on the left and right sides are fixed with a phase difference of $90^{\circ}$ on the rotational axis of the revolute joint of each phalanx. This fixing method facilitates extension/flexion motions by which the tendons on the left side (green lines in Figure 3) pull the joint frames in flexion motion, while the tendons on the right side (red lines in Figure 3) pull the frames in extension motion. Moreover, because the slack and taut problem associated with the tendon-pulley driving method causes poor power transmission, it should be solved for driving the finger module consistently. In this study, to solve this problem within the limited design space, the paths of all tendons passing the PIP and MCP joints were designed to pass 
through the centers of the rotational axes of these joints. This path design facilitates steady power transfer to the joint frames, regardless of the motions of the phalanxes, as illustrated in the upper and side diagrams in Figure 3. This design method has been proven to be considerably efficient in a limited design space, and it decreases product weight because incidental mechanical parts, such as pulleys and shafts, are not needed for driving. In addition, it simplifies the design owing to the use of a minimum number of parts, improves productivity, and reduces production costs.

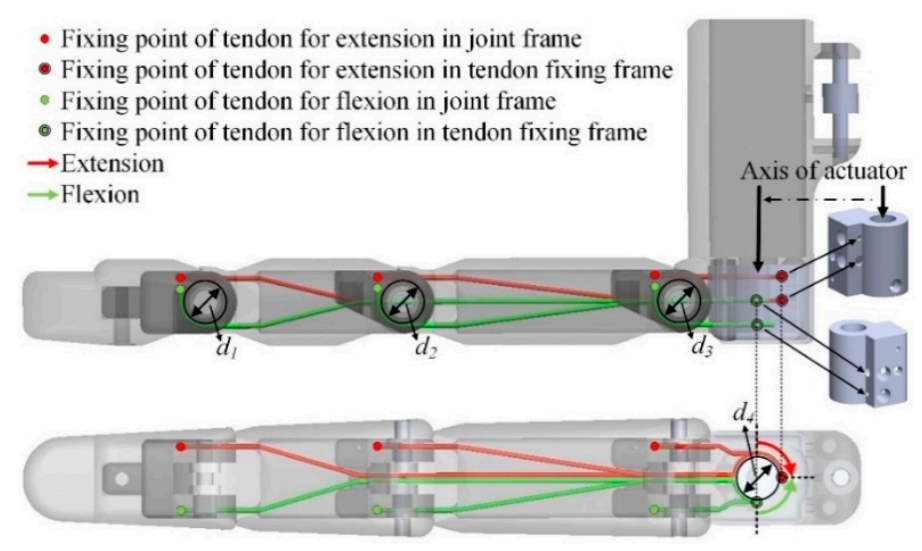

Figure 3. Connecting points and paths of tendons.

The diameters $\left(d_{i}(i=1,2\right.$, and 3$)$ and $\left.d_{4}\right)$ of the joint frames in each phalanx and the tendon-fixing frame were designed equally, i.e., $d_{1}=d_{2}=d_{3}=d_{4}$. Therefore, extension/flexion motions can be realized by driving the actuator in the range of $0^{\circ}-90^{\circ}$.

In the proposed finger module, self-adaptive grasping was achieved using two linear springs in the middle and proximal phalanxes (Figure $4 \mathrm{a}, \mathrm{b}$ ) and a torsional spring in the mounting part (Figure 4c). Specifically, the torsional springs of the four fingers generate adduction/abduction motions passively. As shown in Figure 4a,b, during natural motion in which external forces are absent, each phalanx is driven from $0^{\circ}$ to $90^{\circ}$. However, when the middle and proximal phalanxes are in contact with an object, although the joint frames are rotated continually, the frames are stopped, and the linear springs are compressed. Similarly, the torsional springs are compressed depending on the external forces generated by contact with an object. The torsional spring in the index finger is compressed in the counterclockwise direction, while the other springs are compressed in the clockwise direction (Figure 4c).

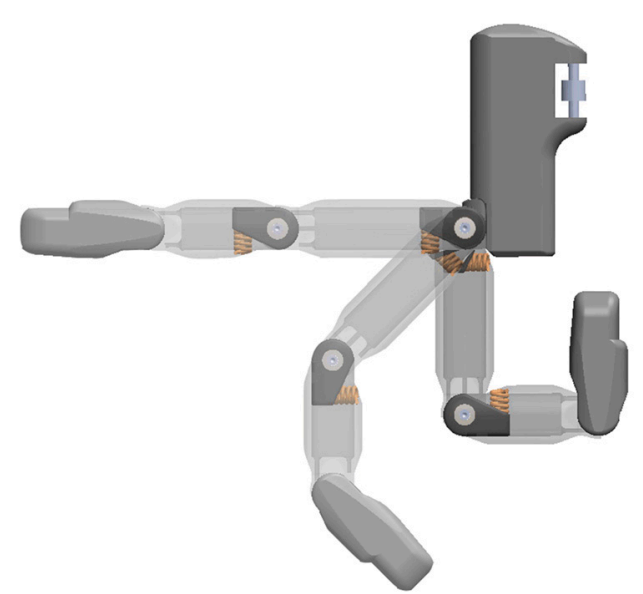

(a)

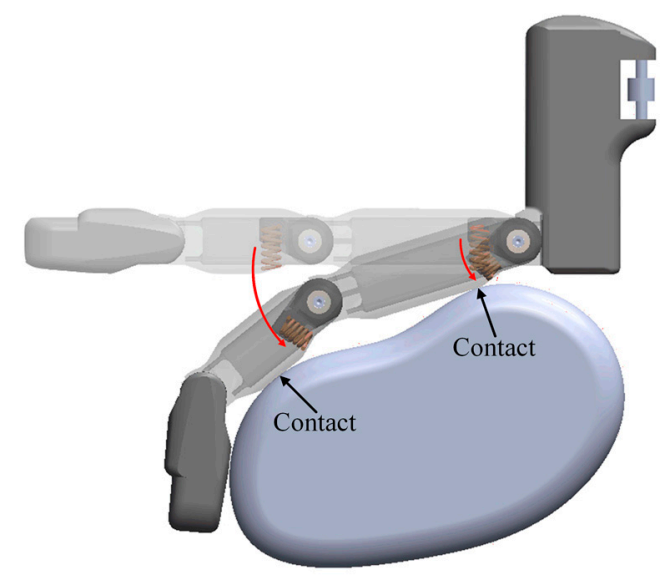

(b)

Figure 4. Cont. 


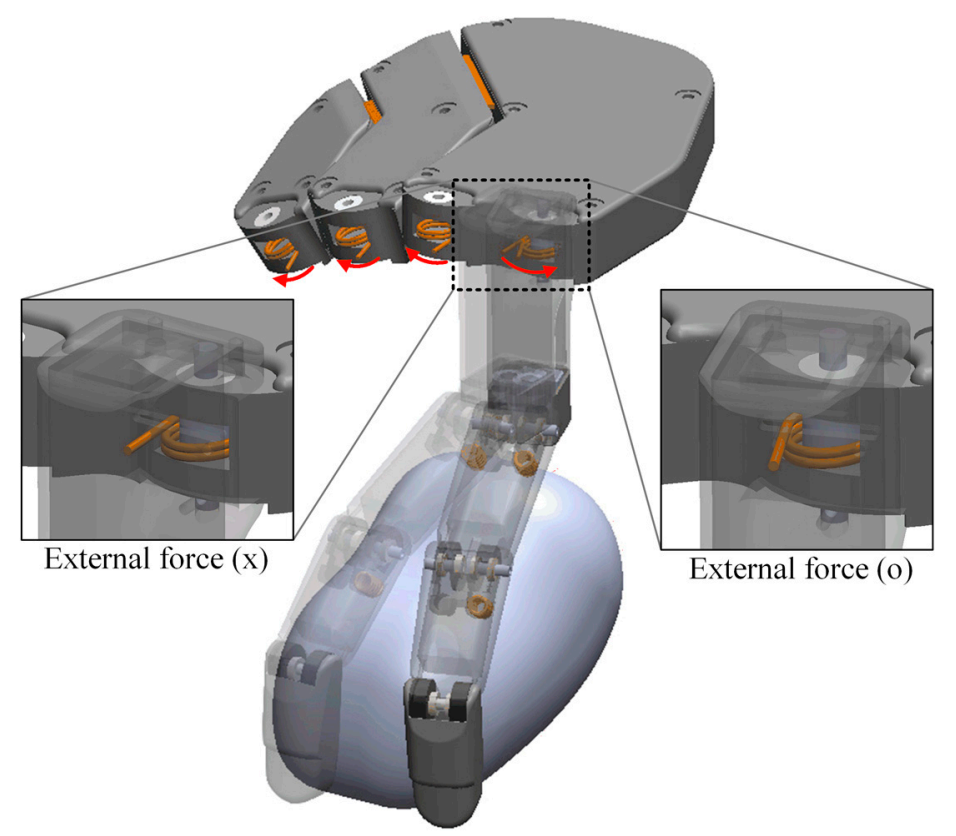

(c)

Figure 4. (a) Natural and self-adaptive grasping motions (b) using two linear springs in the proximal interphalangeal (PIP) and metacarpophalangeal (MCP) joints and (c) using two linear springs and one torsional spring in the PIP and MCP joints.

The finger module and mounting part are composed of 32 and 23 parts, respectively, including the springs, bearings, and shafts, as depicted in Figure 5. The developed prosthetic hand can be assembled easily under the following assembly directions, as suggested in Figure 5.

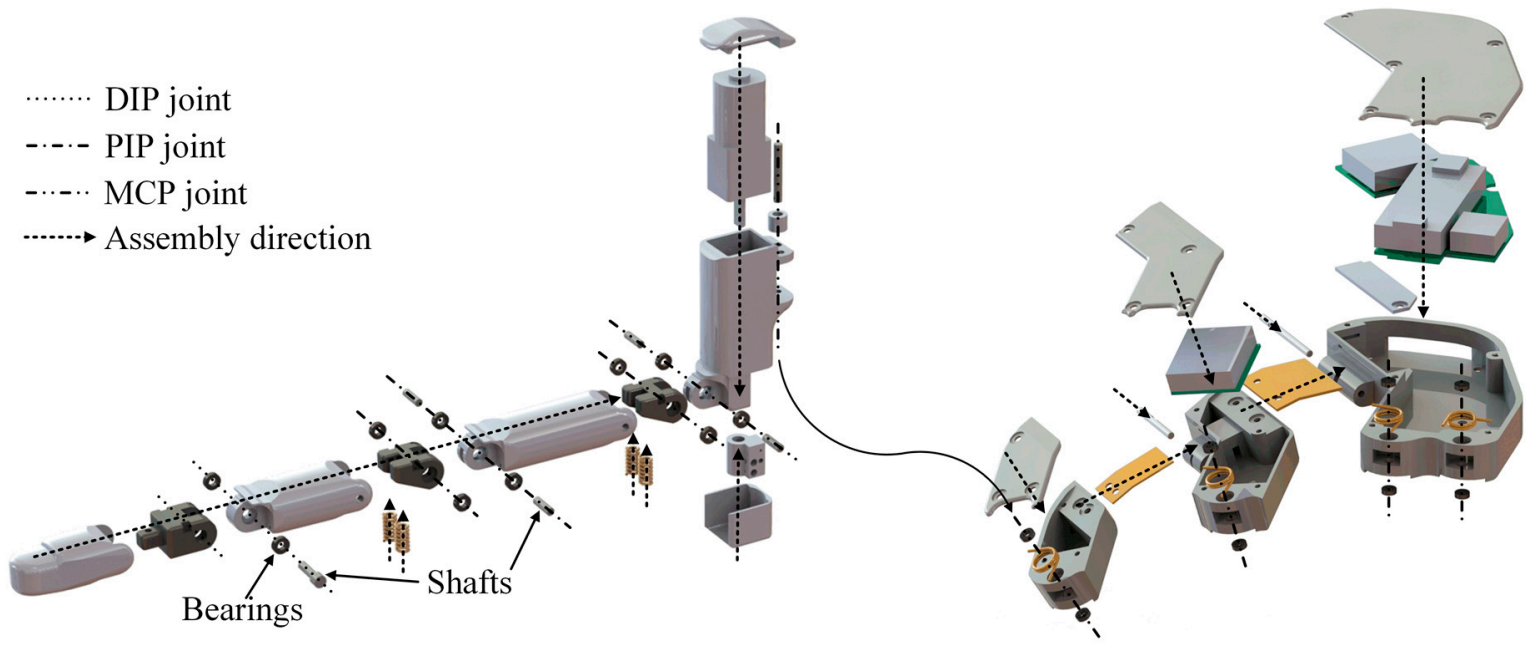

Figure 5. Assembly schematic diagram and all mechanical parts in the prosthetic hand.

\subsection{Control System}

To drive the prosthetic hand, the user wears an operating part on his or her thumb, as illustrated in Figure 6a. The control system consists of an MCU (Arduino Nano), two dual-motor drives (TB6612FNG), two gyro sensors (MPU-6500), and a switch for controlling four actuators. The switch and one gyro sensor are attached to the operating part, and the other components are installed in the mounting part. 


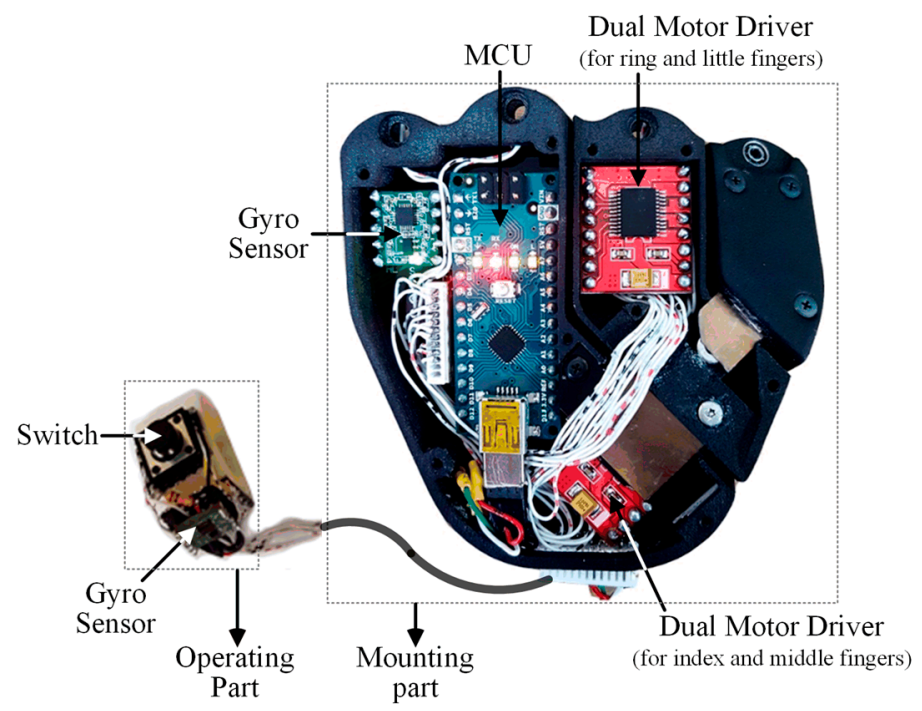

(a)

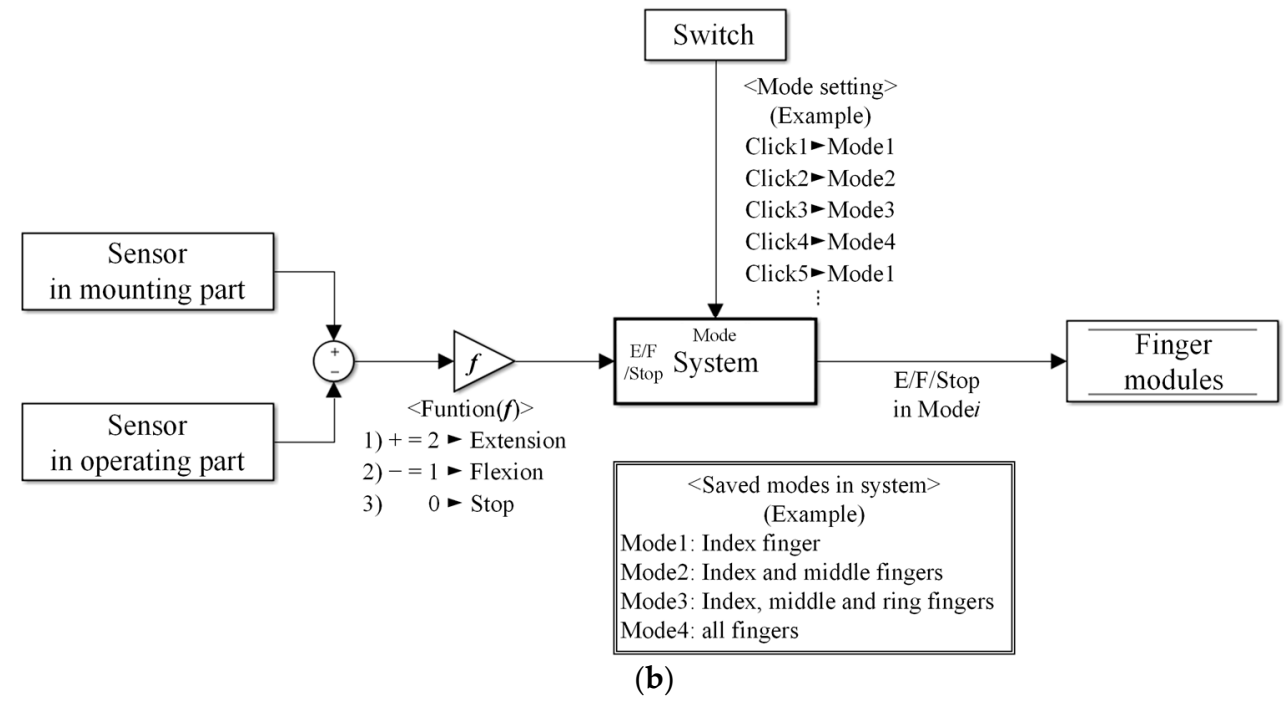

Figure 6. (a) Electrical components in the mounting and operating parts and (b) control schematic diagram.

Grasping is the main target motion. Four grasping patterns are preprogrammed in the controller, and the pattern mode is selected by users with a simple switch. In mode 1, only the index finger moves. In mode 2 , the index and middle fingers move together. In mode 3 , the ring finger moves together with the index and middle fingers. Finally, all fingers move simultaneously in mode 4 . Of course, different combinations can be easily added to the controller to comply with users' needs. With these various patterns, the user can select the desired grasping motion depending on the environment. In each mode, the finger motion is initiated according to the movement of the thumb. In other words, the thumb is used to give the "start" command to the controller. The movement of the thumb is estimated from two gyro sensors: one is attached to the operating part, and the other is in the mounting part. By using the relative value, when the hand is moved without thumb movement, the individual finger modules are not moved, whereas the finger modules are moved when the thumb is moved and the direction of rotation of the actuators is decided depending on the signs of the measured relative values. We control each finger motor at its current mode control. The control concept and schematic diagram are shown in Figure $6 b$. 


\section{Analysis of the Prosthetic Hand}

\subsection{Kinematic Analysis}

\subsubsection{Position Analysis}

Because the diameter of the tendon-fixing frame ( $d_{4}$ in Figure 2$)$ is equal to the diameters of the joint frames in the DIP, PIP, and MCP joints, in natural motion, the angles of each of the phalanxes $\left(\theta_{i}, i=1,2\right.$, and 3$)$ are changed by an identical magnitude $\theta$, i.e., $\theta_{1}=\theta_{2}=\theta_{3}=\theta$, as shown in Figure 7a. If $(X, Y)$ represents the $X$ - and $Y$-coordinates in the coordinate frame $O$, then the positions of points $p_{1}, p_{2}$, and $p_{3}$ can be determined as follows:

$$
\begin{gathered}
p_{1}=\left(l_{1} \cos \theta_{1}, l_{1} \sin \theta_{1}\right), \\
p_{2}=\left(l_{1} \cos \theta_{1}+l_{2} \cos \left(\theta_{1}+\theta_{2}\right), l_{1} \sin \theta_{1}+l_{2} \sin \left(\theta_{1}+\theta_{2}\right)\right) \text { and } \\
p_{3}=\left(l_{1} \cos \theta_{1}+l_{2} \cos \left(\theta_{1}+\theta_{2}\right)+l_{3} \cos \left(\theta_{1}+\theta_{2}+\theta_{3}\right),\right. \\
\left.l_{1} \sin \theta_{1}+l_{2} \sin \left(\theta_{1}+\theta_{2}\right)+l_{3} \sin \left(\theta_{1}+\theta_{2}+\theta_{3}\right)\right) .
\end{gathered}
$$

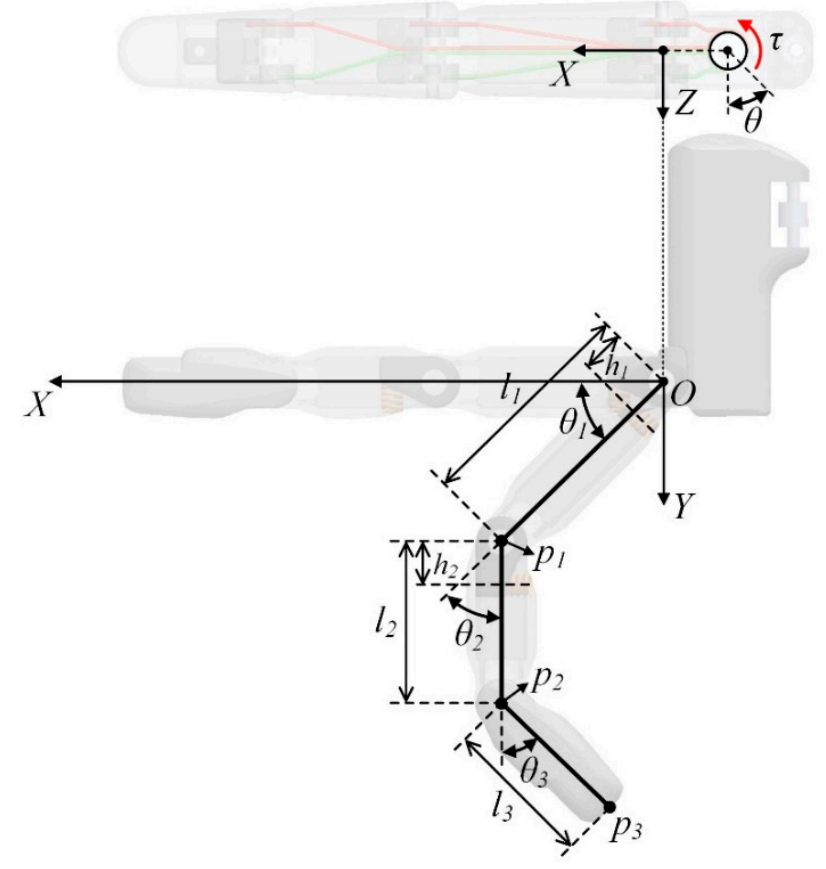

(a)

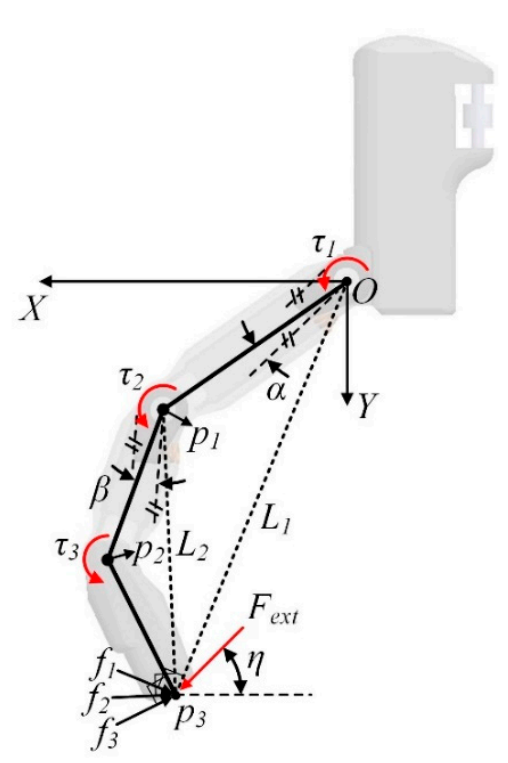

(b)

Figure 7. Coordinate system $(O)$ and variables (a) in natural motion and (b) in a self-adaptive grasping motion.

Because $\alpha$ and $\beta$ (Figure 7b) exist in the self-adaptive grasping motion, the positions of points $p_{1}, p_{2}$, and $p_{3}$ are derived as follows:

$$
\begin{gathered}
p_{1}=\left(l_{1} \cos \left(\theta_{1}-\alpha\right), l_{1} \sin \left(\theta_{1}-\alpha\right)\right), \\
p_{2}=\left(l_{1} \cos \left(\theta_{1}-\alpha\right)+l_{2} \cos \left(\theta_{1}-\alpha+\theta_{2}-\beta\right), l_{1} \sin \left(\theta_{1}-\alpha\right)+l_{2} \sin \left(\theta_{1}-\alpha+\theta_{2}-\beta\right)\right) \text { and } \\
p_{3}=\left(l_{1} \cos \left(\theta_{1}-\alpha\right)+l_{2} \cos \left(\theta_{1}-\alpha+\theta_{2}-\beta\right)\right)+l_{3} \cos \left(\theta_{1}-\alpha+\theta_{2}-\beta+\theta_{3}\right), \\
\left.\left.l_{1} \sin \left(\theta_{1}-\alpha\right)+l_{2} \sin \left(\theta_{1}-\alpha+\theta_{2}-\beta\right)\right)+l_{3} \sin \left(\theta_{1}-\alpha+\theta_{2}-\beta+\theta_{3}\right)\right) .
\end{gathered}
$$




\subsubsection{Velocity Analysis}

When the angular velocity of the actuator during natural motion is $\omega$, the velocities of the points $p_{1}, p_{2}$, and $p_{3}$ are calculated as follows:

$$
\begin{gathered}
V_{1}=\omega\left(-l_{1} \sin \theta, l_{1} \cos \theta\right), \\
V_{2}=\omega\left(-l_{1} \sin \theta-2 l_{2} \sin 2 \theta, l_{1} \cos \theta+2 l_{2} \cos 2 \theta\right) \text { and } \\
V_{3}=\omega\left(-l_{1} \sin \theta-2 l_{2} \sin 2 \theta-3 l_{3} \sin 3 \theta, l_{1} \cos \theta+2 l_{2} \cos 2 \theta+3 l_{3} \cos 3 \theta\right),
\end{gathered}
$$

where $\theta=\theta_{1}=\theta_{2}=\theta_{3}$.

\subsection{Static Analysis}

When the torque $\tau$ produced by an actuator is divided into each joint equally, i.e., $\tau_{1}=\tau_{2}=\tau_{3}=\frac{\tau}{3}$ in Figure 7 , the relation between the forces $\left(f_{i}\right.$ in Figure $\left.7 \mathrm{~b}\right)$ produced by each joint and the external force $\boldsymbol{F}_{\text {ext }}$ generated by contact with an object at point $p_{3}$ can be analyzed according to the ranges of $\alpha$ and $\beta$. The magnitude of the spring force, $f_{s_{j}}(j=1$ and 2$)$, produced by the torques $\left(\tau_{1}\right.$ and $\left.\tau_{2}\right)$ after an object is contacted at $p_{3}$ is derived as follows

$$
f_{s_{j}}=k_{j} x_{j}
$$

where $k_{j}$ and $x_{j}$ are the spring constant and compressed length of the $j$ th linear spring, respectively. For the ranges of $0<\alpha$ and $\beta<25$, the produced forces $\left(f_{1}\right.$ and $\left.f_{2}\right)$ are determined by the spring forces. After the compression of the springs ( $\alpha$ and $\beta=25$ ) is completed, $f_{j}$ are determined by the sum of each spring force and the force produced by $\tau_{j}$. However, the force from the DIP joint is produced by $\tau_{3}$ regardless of self-adaptive grasping. Therefore, the relation between the forces by joints and the external force is derived by

$$
\begin{gathered}
\boldsymbol{F}_{\text {ext }}=\sum_{i=1}^{3} f_{i}=\left[\frac{\tau_{3}}{l_{3}} \cos \theta_{f_{3}}+h_{2} \frac{f_{s_{2}}}{L_{2}} \cos \theta_{f_{2}}+h_{1} \frac{f_{s_{1}}}{L_{1}} \cos \theta_{f_{1}}, \frac{\tau_{3}}{l_{3}} \sin \theta_{f_{3}}+h_{2} \frac{f_{s_{2}}}{L_{2}} \sin \theta_{f_{2}}+h_{1} \frac{f_{s_{1}}}{L_{1}} \sin \theta_{f_{1}}\right]^{T} \\
(\text { for } 0<\alpha \text { and } \beta<25) \text { and } \\
\boldsymbol{F}_{\text {ext }}=\sum_{i=1}^{3} f_{i}=\left[\frac{\tau_{3}}{l_{3}} \cos \theta_{f_{3}}+\frac{\tau_{2}}{L_{2}} \cos \theta_{f_{2}}+\frac{\tau_{1}}{L_{1}} \cos \theta_{f_{1}}+h_{2} \frac{f_{s_{2}}}{L_{2}} \cos \theta_{f_{2}}+h_{1} \frac{f_{s_{1}}}{L_{1}} \cos \theta_{f_{1}},\right. \\
\left.\frac{\tau_{3}}{l_{3}} \sin \theta_{f_{3}}+\frac{\tau_{2}}{L_{2}} \sin \theta_{f_{2}}+\frac{\tau_{1}}{L_{1}} \sin \theta_{f_{1}}+h_{2} \frac{f_{s_{2}}}{L_{2}} \sin \theta_{f_{2}}+h_{1} \frac{f_{s_{1}}}{L_{1}} \sin \theta_{f_{1}}\right]^{T} \\
\text { (for } \alpha \text { and } \beta=25),
\end{gathered}
$$

where $\theta_{f_{i}}$ is the angle between the $X$-coordinate and the $i$ th force, and $h_{j}$ and $L_{j}$ are the distances between the axis of each revolute joint and the $j$ th linear spring and the fingertip $p_{3}$, respectively, as shown in Figure 7b. In Equations (11) and (12), the external force is calculated as follows:

$$
\boldsymbol{F}_{\text {ext }}=\left[f_{\text {ext }} \cos \eta, f_{\text {ext }} \sin \eta\right]^{T},
$$

where $f_{\text {ext }}$ is the magnitude of the external force, and $\eta$ is the angle between the $X$-coordinate and the external force. By using Equations (11), (12), and (13), the specifications of the actuator and gear ratio of the gearbox can be calculated when the derived payload and velocity of the finger module are given.

\subsection{FEM Analysis}

To validate the design and safety for the frame shapes and thicknesses under desired conditions, the finger module is analyzed for stress distribution and deflection using the finite element method (FEM). The simulations set the mechanical parts (shafts and bearings) and the other frames to stainless 
steel and nylon, respectively. Because the magnitude of the external force applied to the finger module is the largest after finishing the adaptive grasping motion, the finger module configuration analyzed is the one shown in Figure $8, \theta=90$, and $\alpha, \beta=25$. Furthermore, Figure 8a shows the result when an external force of $7.5 \mathrm{~N}$ was applied at the tip of the finger module along the $Y$-coordinate, while Figure $8 \mathrm{~b}$ shows the result when an external force of equal magnitude was applied at the middle phalanx along the $X$-coordinate. The magnitude of the external forces is sufficient to validate the design because the target payload in a finger module is $250 \mathrm{~g}$, implying a safety factor of 3 .
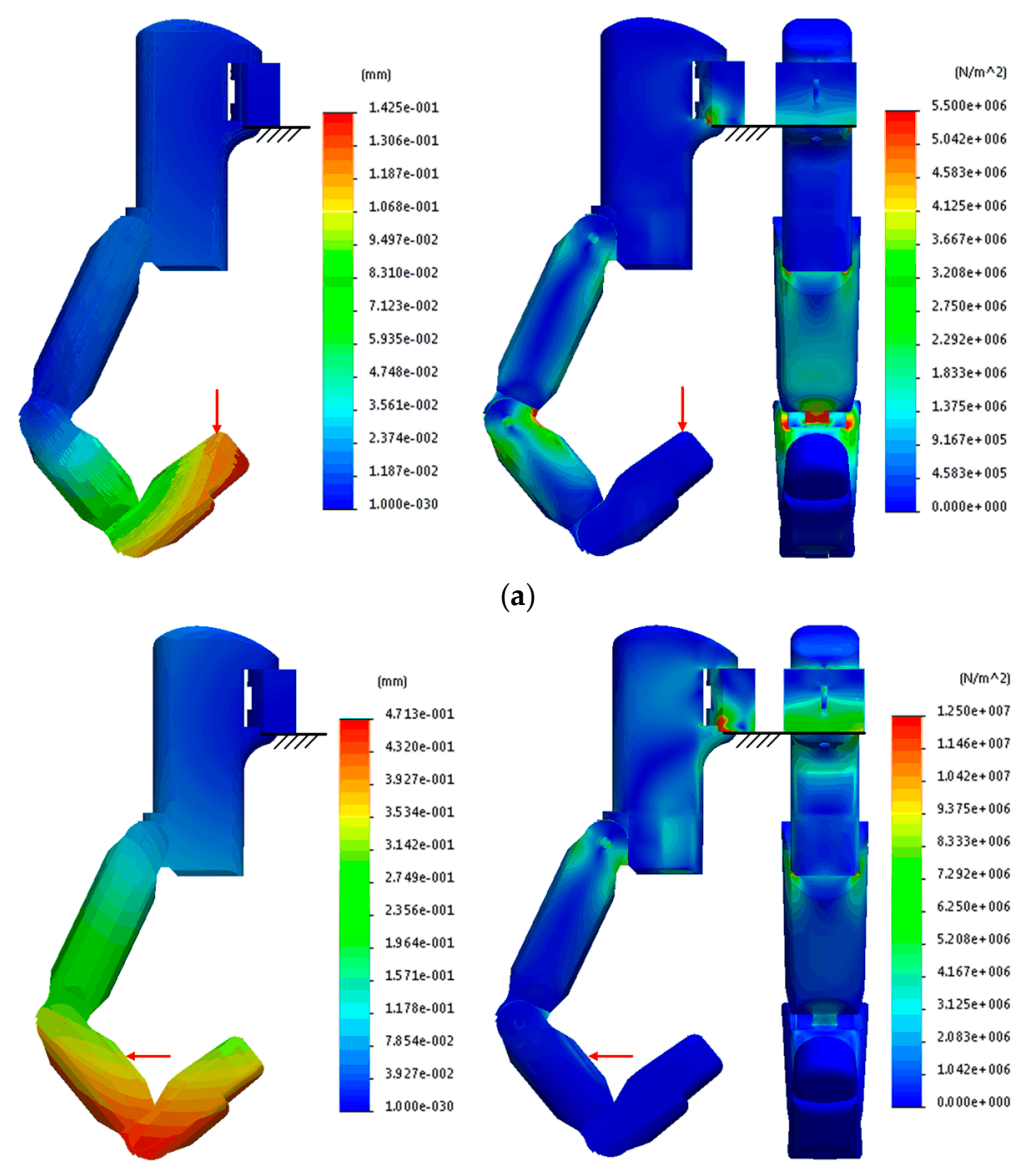

(a)

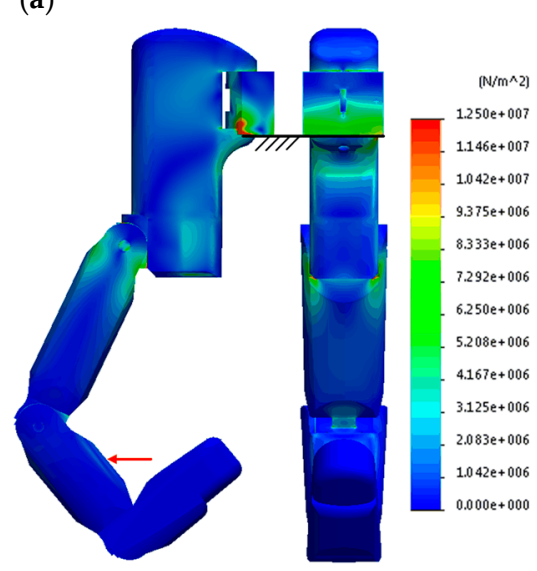

(b)

Figure 8. Deflection (left) and stress distribution (right) when external forces were applied along (a) $Y$ and (b) X-coordinates, respectively.

The maximum deflections in Figure $8 \mathrm{a}, \mathrm{b}$ were $0.143 \mathrm{~mm}$ and $0.471 \mathrm{~mm}$, and the maximum generated stresses were in the middle phalanx $\left(51.484 \times 10^{6} \mathrm{~N} / \mathrm{m}^{2}\right)$ and the palm $\left(112.059 \times 10^{6} \mathrm{~N} / \mathrm{m}^{2}\right)$, respectively. Thus, the developed prosthetic hand can perform the desired motions suitably. Because the middle phalanx and palm are made of nylon (tensile strength of nylon is $139.043 \mathrm{MPa}$ ), the prosthetic hand has adequate rigidity in the desired conditions.

\section{Results}

\subsection{Prototype}

The prototype was composed of the prosthetic hand and operating part, as shown in Figure 1a. All parts except the shafts were manufactured from nylon using the MJF 3D printing, and the shafts were made of stainless steel. To satisfy the design conditions, the linear, torsional, and leaf springs adhered to the following specifications. 
(1) The available range of linear springs installed on the joint frames was $4 \mathrm{~mm}$, the spring constant was $2.667 \mathrm{~N} / \mathrm{mm}$, and the length for preload applied by frames was $1 \mathrm{~mm}$,

(2) The available range of torsional springs was $20^{\circ}$, the diameter and internal diameter of the coil and torsional spring were $1 \mathrm{~mm}$ and $5 \mathrm{~mm}$, respectively, and the angle for preload was $5^{\circ}$, and

(3) The available range of leaf spring was $20^{\circ}$, the thickness of leaf springs was $0.7 \mathrm{~mm}$, and the lengths of the leaf springs were $10 \mathrm{~mm}$ and $20 \mathrm{~mm}$, respectively.

The diameter of the tendon was $0.6 \mathrm{~mm}$, and the allowable load was $6 \mathrm{~kg}$. Furthermore, the actuator has a gearbox with a 986.41:1 ratio and $31 \mathrm{rpm}$. The mounting part is installed on the back of the patient's hand, and the arrangement between the palm of the patient and the fingers of the prosthetic hand can be adjusted adequately. The weight and product cost of the prototype were $152.32 \mathrm{~g}$ and 1246 USD, respectively. The cost included 3D printing (460 USD), machining (403 USD), mechanical parts (333 USD), and electrical parts (50 USD). Because of the use of the actuator with $31 \mathrm{rpm}$, each phalanx moves $185.8^{\circ} / \mathrm{s}$. When the applied external force is parallel to the $X$-coordinate $(\eta=0$ in Equation (13)), and the variables in Equation (12) are $h_{1}=h_{2}=6.5 \mathrm{~mm}, \theta=45$, and $\alpha=\beta=25$, one finger module is capable of lifting the object with $0.609 \mathrm{~kg}$ because the output torque from the gearbox is $210 \mathrm{Nmm}$. In other words, using four finger modules, the prosthetic hand can raise over $2.435 \mathrm{~kg}$ at the fingertip in these conditions. The specifications of the developed prosthetic hand are summarized in Table 2. The developed prosthetic hand is lighter than the previous models, and its size is similar to the average size of American female hands. The grasp speed $\left(185.8^{\circ} / \mathrm{s}\right)$ exceeded the minimal acceptable speed $\left(115^{\circ} / \mathrm{s}\right)$ suggested in [13].

Table 2. Specifications of the developed prosthetic hand.

\begin{tabular}{|c|c|c|c|c|}
\hline Weight & \multicolumn{4}{|c|}{$152.32 \mathrm{~g}$ (Except thumb) } \\
\hline Overall size [length, width, thickness] & \multicolumn{4}{|c|}{$[174 \mathrm{~mm}, 77 \mathrm{~mm}, 11 \mathrm{~mm}]$} \\
\hline \multirow{4}{*}{ Finger sizes [length, width, thickness] } & \multicolumn{2}{|c|}{ Index finger } & \multicolumn{2}{|c|}{$[83.7 \mathrm{~mm}, 14.8 \mathrm{~mm}, 11 \mathrm{~mm}]$} \\
\hline & \multicolumn{2}{|c|}{ Middle finger } & \multicolumn{2}{|c|}{$[88.7 \mathrm{~mm}, 14.8 \mathrm{~mm}, 11 \mathrm{~mm}$} \\
\hline & \multicolumn{2}{|c|}{ Ring finger } & \multicolumn{2}{|c|}{$[83.7 \mathrm{~mm}, 14.8 \mathrm{~mm}, 11 \mathrm{~mm}$} \\
\hline & \multicolumn{2}{|c|}{ Little finger } & \multicolumn{2}{|c|}{$[73.7 \mathrm{~mm}, 14.8 \mathrm{~mm}, 11 \mathrm{~mm}]$} \\
\hline Degrees of freedom & \multicolumn{4}{|c|}{18} \\
\hline Active motions (The number of actuators) & \multicolumn{4}{|c|}{4} \\
\hline Passive motions & \multicolumn{4}{|c|}{$14^{*}$} \\
\hline Joint coupling method & \multicolumn{4}{|c|}{ Tendon } \\
\hline Adaptive grasping & \multicolumn{4}{|c|}{ Yes } \\
\hline Grip Forces & \multicolumn{4}{|c|}{$\begin{array}{l}\text { Maximum force: } 6.460 \mathrm{~N} \text { (at } \eta=0 \text { ) and } 7.487 \mathrm{~N} \text { (at } \eta=90) \\
\quad(\text { for } 0 \leq \theta \leq 65 \text { and } \alpha=\beta=25 \text { in a finger module) }\end{array}$} \\
\hline \multirow{6}{*}{ Ranges of Motion } & \multirow{3}{*}{ Extension/Flexion } & \multicolumn{2}{|c|}{ DIP joint } & $0-90$ \\
\hline & & \multicolumn{2}{|c|}{ PIP joint } & $0-90$ \\
\hline & & \multicolumn{2}{|c|}{ MCP joint } & $0-90$ \\
\hline & \multicolumn{3}{|c|}{ Adduction/Abduction } & $0-15$ \\
\hline & \multicolumn{3}{|c|}{ Adaptive grasping in PIP and MCP joints } & $0-25$ \\
\hline & \multicolumn{3}{|c|}{ Palm } & $0-30$ \\
\hline Finger/Grasp speed & \multicolumn{4}{|c|}{ Up to $185.8^{\circ} / \mathrm{s}$ at each phalanx } \\
\hline
\end{tabular}

* The number of passive motions realized using linear springs: 8 , torsional springs: 4, and leaf springs: 2 . DIP: the distal interphalangeal, PIP: the proximal interphalangeal, and MCP: the metacarpophalangeal.

\subsection{Experiment}

The experiment for performance verification of the prosthetic hand involved passive and active hand motions. Passive motions included palm movement and adduction/abduction motions. First, 
passive motions were executed. Figure $9 \mathrm{a}, \mathrm{b}$ show the palm movement and adduction/abduction motions, respectively. The initial configuration of the mounting part was set to $15^{\circ}$ by leaf springs at each joint, as shown in Figures $2 \mathrm{~b}$ and $9 \mathrm{a}$. Furthermore, in the adduction/abduction motions, the index finger was rotated counterclockwise, while the other fingers (middle, ring, and little fingers) were rotated clockwise in the range from $0^{\circ}$ to $15^{\circ}$ (Figure $9 \mathrm{~b}$ ).
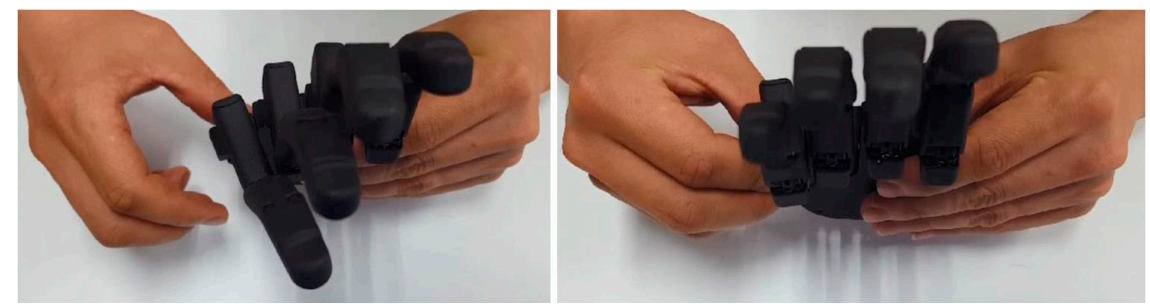

(a)
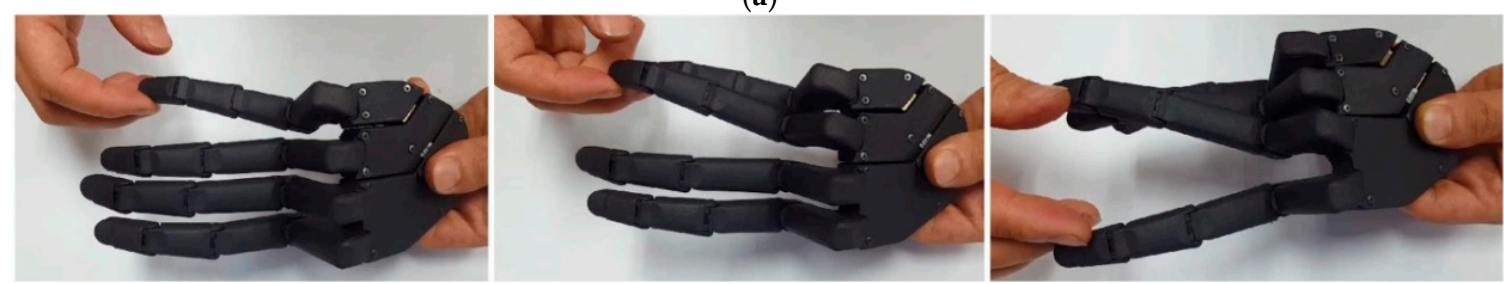

(b)

Figure 9. Passive motions in (a) palm movements and (b) adduction/abduction motions.

Second, the practical applicability of the developed prosthetic hand was verified using diverse objects encountered in daily life. Objective motions, such as natural motion and self-adaptive grasping, were tested (Figure 10). In natural motion, the prototype was moved, as shown in Figure 10a. Because of the absence of external forces, each phalanx moved from $0^{\circ}$ to $90^{\circ}$. In addition, because the initial configuration of the palm was set to $15^{\circ}$ at the two joints, slight interference was observed between the middle and ring fingers in the final configuration of natural motion (the red circle in Figure 10a). Self-adaptive grasping was tested with diverse objects (Figure 10b). In particular, the four fingers of the developed hand could maintain the grasping configuration while lifting the $1.36 \mathrm{~kg}$ dumb-bell. In all experiments, the developed prosthetic hand robustly grasped various objects.

All motions including passive and self-adaptive grasping motions can be checked in supplementary video, and it is guided in Supplementary Materials.
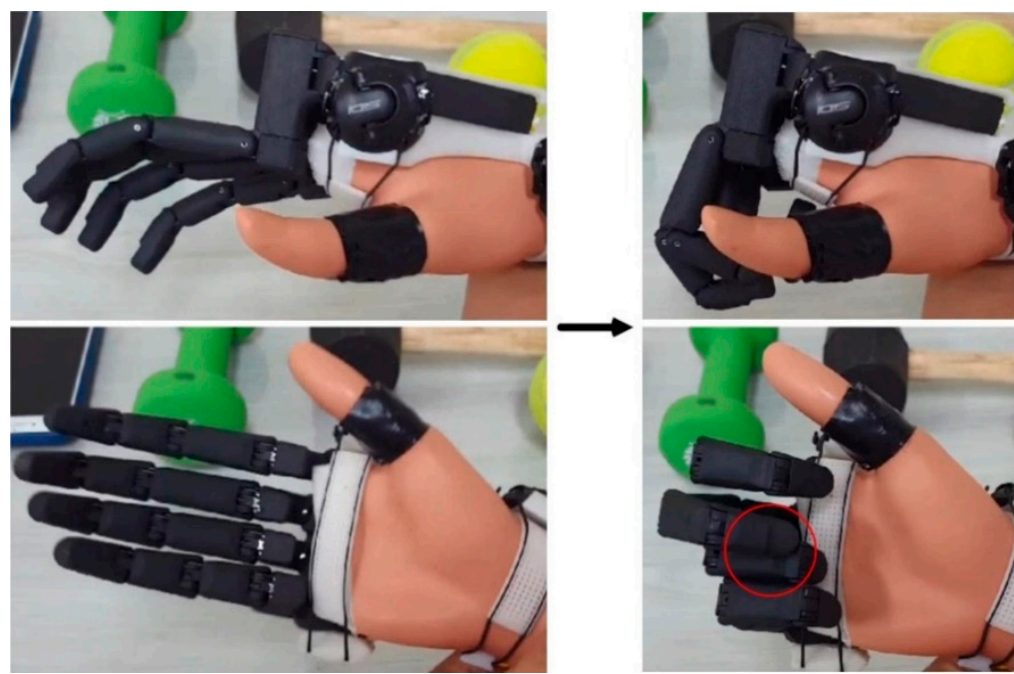

(a)

Figure 10. Cont. 


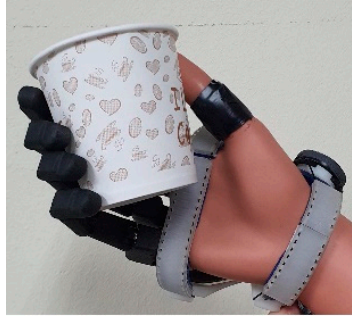

Paper cup

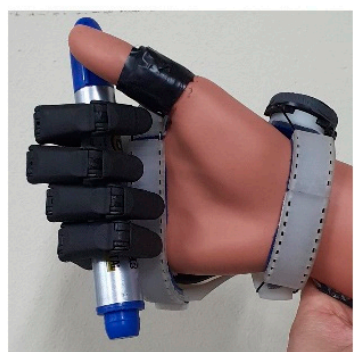

Pen

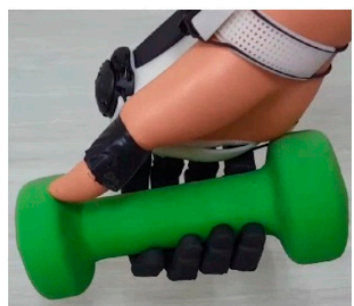

Dumb-bell

$(1.36 \mathrm{~kg})$

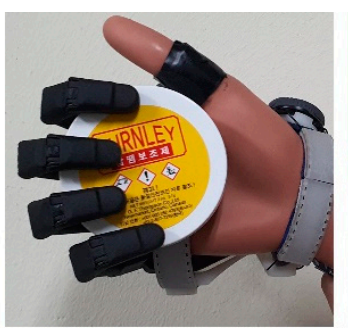

Cylindrical box

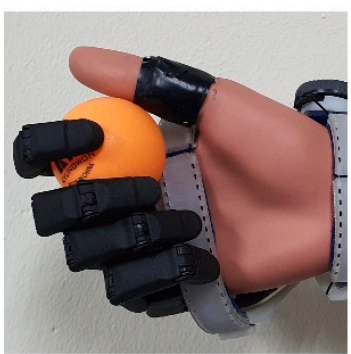

Ping-pong ball

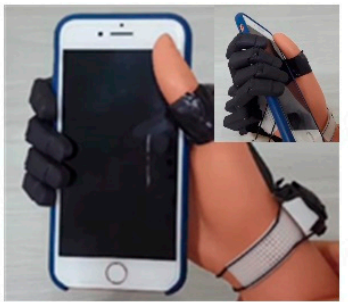

Smart-phone

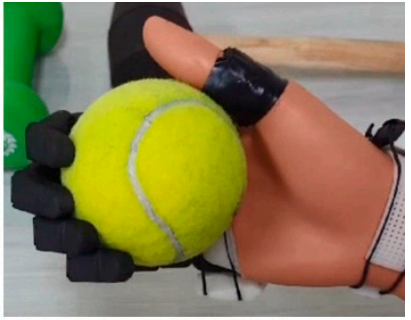

Tennis ball

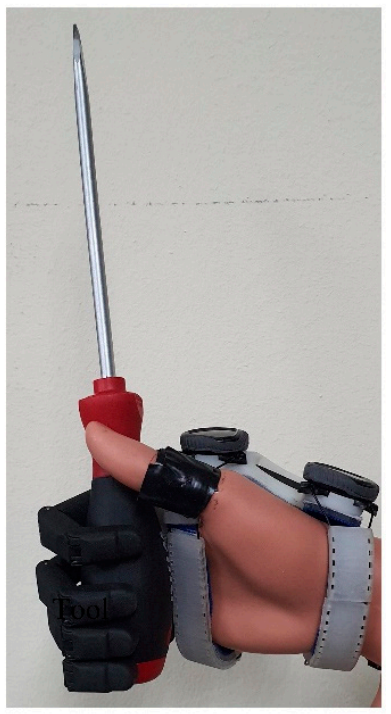

Tool

(b)

Figure 10. (a) Natural and (b) self-adaptive grasping motions with diverse objects.

\section{Conclusions}

We developed the lightweight prosthetic hand for amputees who retain their thumb and partial metacarpal. To accommodate the actuators and electrical components within the limited design space, the actuators are installed vertically between the finger modules and the mounting part. Furthermore, for weight reduction, all frames were manufactured from nylon using the MJF 3D printing, except for the shafts. Each prosthetic finger has 4-DOFs with one active and three passive motions. The DIP, PIP, and MCP joints in each finger module are driven using tendons and an actuator. Functions, such as self-adaptive grasping, extension/flexion, and adduction/abduction motions, were achieved, and passive motions were realized using linear and torsional springs. Finally, the performance of the prosthetic hand was verified experimentally, and its weight $(152.32 \mathrm{~g})$ is considerably lower than that of conventional prosthetic hands.

Supplementary Materials: The following are available online at http://www.mdpi.com/2076-3417/10/10/3536/s1, Video S1: title: A lightweight prosthetic hand.

Author Contributions: Conceptualization, W.R. and Y.C.; Methodology, W.R. and S.L.; Software, W.R. and Y.C.; Formal analysis, W.R. and Y.J.C.; Writing-original draft preparation, W.R.; Supervision, Y.C., Y.J.C. and S.L.; Project administration, S.L.; Writing-review and editing, Y.C., Y.J.C. and S.L. All authors have read and agreed to the published version of the manuscript.

Funding: Research was supported in part by the convergence technology development program for bionic arm through the National Research Foundation of Korea (NRF) funded by the Ministry of Science \& ICT (No. 2015M3C1B2052811), Republic of Korea.

Conflicts of Interest: The authors declare no conflict of interest. 


\section{References}

1. Hazani, R.; Buntic, R.F.; Brooks, D. Patterns in blast injuries to the hand. Hand 2009, 4, 44-49. [CrossRef] [PubMed]

2. Venkatramani, H.; Bhardwaj, P.; Sierakowski, A.; Sabapathy, S.R. Functional outcomes of post-traumatic metacarpal hand reconstruction with free toe-to-hand transfer. Indian J. Plast. Surg. 2016, 49, 16-25. [CrossRef] [PubMed]

3. Del Piñal, F. The indications for toe transfer after "minor" finger injuries. J. Hand Surg. 2004, 29, 120-129.

4. Rodriguez, N.E.N.; Carbone, G.; Ceccarelli, M. Optimal design of driving mechanism in a 1-DOF anthropomorphic finger. Mech. Mach. Theory 2006, 41, 897-911. [CrossRef]

5. Yoon, D.C.; Lee, C.; Choi, Y. Underactuated Finger Mechanism for Body-Powered Partial Prosthesis. J. Korea Robot. Soc. 2016, 11, 193-204. [CrossRef]

6. Kamikawa, Y.; Maeno, T. Underactuated Five-Finger Prosthetic Hand Inspired by Grasping Force Distribution of Humans. In Proceedings of the IEEE/RSJ International Conference on Intelligent Robots and Systems, Acropolis Convention Center, Nice, France, 22-26 September 2008.

7. Jing, X.; Yong, X.; Tian, L.; Togo, S.; Jiang, Y.; Yokoi, H.; Li, G. Development of Tendon Driven Under-Actuated Mechanism Applied in an EMG Prosthetic Hand with Three Major Grasps for Daily Life. In Proceedings of the IEEE/RSJ International Conference on Intelligent Robots and Systems (IROS), Madrid, Spain, 1-5 October 2018.

8. Mottard, A.; Laliberte, T.; Gosselin, C. Underactuated tendon-driven robotic/prosthetic hands: Design issues. In Proceedings of the Robotics: Science and Systems, Cambridge, MA, USA, 12-16 July 2017.

9. RSL Steeper Website. Leeds (United Kingdom): RSL Steeper; 2013. Available online: http://rslsteeper.com/ (accessed on 15 April 2020).

10. Touch Bionics Website. Mansfield (MA): Touch Bionics Inc.; 2013. Available online: http://www.touchbionics. $\mathrm{com} /$ (accessed on 17 April 2020).

11. Ryu, W.; Kim, D.; Choi, Y.; Lee, S. Development of Prosthetic Finger with Actuator. In Proceedings of the 16th International Conference on Ubiquitous Robots (UR), Jeju, Korea, 24-27 June 2019.

12. Clement, R.G.E.; Bugler, K.E.; Oliver, C.W. Bionic prosthetic hands: A review of present technology and future aspirations. Surg. J. R. Coll. Surg. Edinb. Irel. 2011, 9, 336-340. [CrossRef] [PubMed]

13. Belter, J.T.; Segil, J.L.; Dollar, A.M.; Weir, R.F. Mechanical design and performance specifications of anthropomorphic prosthetic hands: A review. J. Rehabil. Res. Dev. 2013, 50, 599-618. [CrossRef] [PubMed]

14. Habib, F.N.; Iovenitti, P.; Massod, S.G.; Nikzad, M. Fabrication of polymeric lattice structures for optimum energy absorption using Multi Jet Fusion technology. Mater. Des. 2018, 155, 86-98. [CrossRef]

15. Sillani, F.; Kleijnen, R.G.; Vetterli, M.; Schmid, M.; Wegener, K. Selective laser sintering and multi jet fusion: Process-induced modification of the raw materials and analyses of parts performance. Addit. Manuf. 2019, 27, 32-41. [CrossRef]

16. Pons, J.L.; Rocon, E.; Ceres, R.; Reynaerts, D.; Saro, B.; Kevin, S.; Van Moorleghem, W. The MANUS-HAND* Dextrous Robotics Upper Limb Prosthesis: Mechanical and Manipulation Aspects. Auton. Robot. 2004, 16, 143-163. [CrossRef]

17. Anthropometry and Biomechanics. Available online: https://msis.jsc.nasa.gov/sections/section03.htm (accessed on 17 April 2020).

(C) 2020 by the authors. Licensee MDPI, Basel, Switzerland. This article is an open access article distributed under the terms and conditions of the Creative Commons Attribution (CC BY) license (http://creativecommons.org/licenses/by/4.0/). 